\title{
Management of pain in oral lichen planus patients: a comparative pilot study
}

\author{
Hanaa M El Shenawy, Amany Mohy Eldin and Sherine A Nasry * (D)
}

\begin{abstract}
Background: Treating oral lichen planus (OLP) is a big challenge for clinicians. Despite numerous existing remedies, to date, no effective cure has been found, which is mainly attributed to the lack of understanding of the pathogenesis of the disease. Our aim was to compare the effectiveness of topical steroids and diode laser in treating OLP patients. Twenty-four patients with OLP were allocated into two groups of 12 patients each. One group was treated with a 970$\mathrm{nm}$ diode laser applied twice weekly and the other group was treated with topical steroids applied four times per day. Patients were followed up for 2 months. Pain was recorded using the visual analog scale (VAS) and the clinical signs and symptoms were recorded using the reticular, atrophic, erosive (RAE) sores. Pain and RAE records were taken before treatment, after treatment, and after 2 months.
\end{abstract}

Results: When pain and RAE scores were compared before and after treatment, a significant lower pain score $(P=0$. 020) and RAE scores $(P=0.025)$ were observed in the steroid group than the laser group. No significant differences in pain $(P=0.333)$ and RAE $(P=0.06)$ scores were observed between both groups in the follow-up. No significant difference was seen between the improvement parameters in the two groups $(P=0.117 \%)$, although better scores were seen in the steroid group.

Conclusions: Topical steroids reduce pain and RAE scores in OLP patients more than laser treatment.

Trial registration: NCT03572959, Registration date: 30th June 2018, retrospectively registered

Keywords: Corticosteroids, Low-level laser, Lichen planus, Pain

\section{Background}

Oral lichen planus (OLP) is a chronic mucocutaneous disorder of unknown etiology with a reported prevalence of 1 to $2.0 \%$ (average 1.27\%) in different countries and is more common in females aged $30-60$ years with a $1.57 \%$ frequency in women compared to $0.96 \%$ in men (McCartan and Healy 2008; Gupta and Jawanda 2015). OLP can present itself in six clinical presentations, mainly the reticular, erosive, atrophic, plaquelike, papular, and bullous types (Canto et al. 2010). The erosive, atrophic, and bullous forms are symptomatic and necessitate treatment. However, despite numerous existing remedies, to date, no effective cure has been found, which is mainly attributed to the lack of understanding of the pathogenesis of the disease (Kaplan et al. 2012). Nevertheless, corticosteroids remain the

\footnotetext{
* Correspondence: nasrysherine@yahoo.com

Surgery and Oral Medicine Department, Oral and Dental Research Division, National Research Centre, P.O.B: 12622, 33 El Buhouth St., Ad Doqi, Dokki, Cairo Governorate, Egypt
}

primarily used medications for the treatment of symptomatic OLP. Among the topical corticosteroids, triamcinolone acetonide paste is the most widely used topical medication for OLP (Arunkumar et al. 2015). However, in many cases, the side effects of long-term treatment with corticosteroid are not tolerated by many patients, or patients are not comfortable with this kind of treatment and are unable to comply with it, requiring the search for alternative efficient therapy (Sousa and Rosa 2008).

Therapeutic laser treatment, known as low-level laser therapy (LLLT) offers many benefits. In addition to being a non-invasive and sterile treatment, it has a biostimulatory effect as it promotes healing of tissue and reduces edema, inflammation, and pain (Agha-Hosseini et al. 2012). It therefore represents an alternative treatment to painful inflammatory oral disorders, especially a chronic disease which requires long-term therapy or to which patients are resistant (Aggarwal et al. 2014). 
Several low-level lasers have been used for treating oral lichen planus lesions including ultraviolet waves, helium-neon, and more recently diode laser, which has many advantages over other lasers because of its smaller size and wide range of spectrum that enables it to be applied in many medical fields (Jin et al. 2010).

In a previous clinical trial, 13 OLP patients who were resistant to standard therapy responded well to LLLT where significant reduction in pain and lesions' size was reported with no side effects or complications observed in any of the patients treated (Cafaro et al. 2010). Another study comparing the effect of LLLT with topical corticosteroids in the treatment of atrophic/erosive OLP demonstrated reduction in pain and appearance scores, as well as lesions' severity in both groups with no significant differences found between the two groups (HH et al. 2011). Patients' compliance and satisfaction to LLLT without compromising health and function as well as the ease of application of laser are other important benefits of this type of therapy (Akbulut et al. 2013).

The aim of this work was to study the efficacy of diode laser in reducing pain and clinical symptoms in OLP patients in comparison to the commonly used triamcinolone acetonide steroid paste.

\section{Methods}

\section{Clinical evaluation}

Twenty-four patients (16 females, 8 males ) were recruited in this study from the Dental Clinic of the National Research Centre, Egypt, and the Ethical Committee approved the study protocol. All patients were informed about the treatment plan and they all submitted a written informed consent before enrolment, which was carried out in accordance with the declaration of Helsinki. Inclusion criteria included oral lesions clinically and histopathologically consistent with the modifications for the WHO 2003 criteria suggested by van der Meij and van der Waal (2003). Patients were excluded from the study in case of indefinite diagnosis (such as lichenoid inflammation), or those suffering from any disease that may present with features similar to OLP (graft versus host disease or lupus erythematosus), if they received any medication for OLP treatment in the 2 months prior enrolment in the study, if they were pregnant or lactating women, or if histological diagnosis revealed lichenoid changes or any signs of dysplasia (Kaplan et al. 2012; Cafaro et al. 2014).

\section{Study design}

Patients were randomly divided into two groups using a randomization software, where a blocked randomization was used to ascertain equal distribution of patients into each group (parallel-group study) (Vickers 2006). One group (12 patients) was irradiated with diode laser and the other group was treated with topical steroids. In the laser group, normal protective measures were taken, where patients and personnel wore laser safety glasses. OLP lesions were irradiated with a 970-nm diode laser (SIRO Laser Advance class III b, SIRONA, Germany) with a $2-\mathrm{W}$ irradiation power in a continuous noncontact mode. The laser beam was delivered using a fiber-optic tip with a $320-\mu \mathrm{m}$ diameter with defocused mode directed at the lesions plus $0.5-\mathrm{cm}$ peri-lesional tissues with a slight overlapping in order to evenly distribute energy covering all the lesional and peri-lesional tissues until blanching of the area was observed (Cafaro et al. 2014). Diode laser was calibrated to an output power of $3 \mathrm{~W}$, frequency of $30 \mathrm{~Hz}$, energy of $180 \mathrm{~J}$, and time interval of 8 min divided into four sessions, $2 \mathrm{~min}$ each with $1 \mathrm{~min}$ rest in between to allow for tissue relaxation.

Irradiation was done twice weekly (once every third day) for 2 months until the resolution of signs (meaning the resolution of all atrophic-erosive lesions, regardless of any persisting hyperkeratotic lesions) for a maximum of ten sessions ( $\mathrm{HH}$ et al. 2011). After each session, patients were advised to have a cold diet and use chlorhexidine oral gel postoperatively. The patients applied the gel twice a day to the lesion for 1 week (Rodríguez-Pérez et al. 2013).

For the steroid group (12 patients), $0.1 \%$ topical triamcinolone acetonide preparation (Kenacourt-A Orabase Pomad, DEVA HOLDINGS A.S., Istanbul, Turkey) was used where the patients were instructed to apply the gel four times daily, with no food or fluid taken $1 \mathrm{~h}$ after application. Patients used the medication for 4 weeks, and if extension of treatment was required after that period, patients were instructed to apply miconazole oral gel (JANSSEN-CILAG Pty Ltd 1-5 Khartoum Road, North Ryde, NSW 2113, Australia) four times a day for 1 week to protect from superimposed fungal infections (Vickers 2006). Scoring of the clinical signs was done according to the RAE (reticular, erosive, atrophic) scale of Thongprasom and co-workers (Thongprasom et al. 1992). Total improvement of the clinical signs was given scores 0 or 1 and was defined as the disappearance of all atrophic-erosive lesions, whether any hyperkeratotic lesions persisted or not. Partial improvement or persisting of the patient's condition meant a decrease (score 2, 3, or 4) or no improvement (no change in the patient's score). Hence, the clinical and symptomatic improvement of the patient's lesion was expressed by the numerical difference between baseline and endpoint scores (Cafaro et al. 2014). Pain was recorded using the visual analog scale (VAS), which consisted of a 10-cm horizontal line, starting from 0 (designating no pain experienced 
by the patient) to 10 (designating unbearable or most severe pain). Pain and RAE records were taken before treatment, after treatment, and then after 2-month follow-up.

\section{Statistical analysis}

Exploration for normality of numerical data was done by checking the distribution of data and calculating the mean and median values as well as using the Kolmogorov-Smirnov and Shapiro-Wilk tests of normality. All data were presented as mean and standard deviation (SD) values. Parametric distribution was seen in the "age" data while "VAS and RAE" data showed non-parametric distribution. For parametric data, Student's $t$ test was used to compare between the two groups, while Mann-Whitney $U$ test was used to compare between the two groups in non-parametric data. The changes after treatment in each group were studied using Friedman's test. If Friedman's test turned significant, pair-wise comparisons were done using the Wilcoxon signed-rank test, and hence, Bonferroni's correction was applied for this comparison. Qualitative data were presented as frequencies $(n)$ and percentages (\%). Chi-square test was used to compare between the two groups. The significance level was set at $P \leq 0.05$. Statistical analysis was performed with $\mathrm{IBM}^{\circ} \mathrm{SPSS}^{\circ}$ Statistics Version 20 for Windows.

\section{Results}

\section{Demographic data}

All 24 symptomatic OLP patients that were recruited in this study continued the treatment protocol till the end of the study. There were 12 patients in each group. The steroid group consisted of 10 females and 2 males with an age range of 45-62 years (mean 52.2), and the laser group consisted of 9 females and 3 males with an age range of 35-70 years and a mean age of 53.6 (Table 1).

There was no statistically significant difference between either mean age values or gender distributions in the two groups ( $P=0.766,0.615$ respectively) (Table 1 ).

Table 1 Descriptive statistics and results of comparison between demographic data in the two groups

\begin{tabular}{lllc}
\hline & Corticosteroids $(n=12)$ & Laser $(n=12)$ & $P$ value \\
\hline Age (mean, SD) & $52.2(6.4)$ & $53.6(13.2)$ & 0.766 \\
Gender $(n, \%)$ & & & \\
Females & $10(83.3)$ & $9(75.0)$ & 0.615 \\
Males & $2(16.7)$ & $3(25.0)$ & \\
\hline
\end{tabular}

Significant at $P \leq 0.05$

\section{Pain (VAS) scores}

Before treatment, no statistically significant difference was found between the two groups $(P$ value $=0.807$ ). Comparison between post-treatment VAS scores resulted in significantly lower mean pain scores in the corticosteroid than in the laser group $(P$ value $=0.020)$, while no statistically significant difference was demonstrated between the two groups in the follow-up period ( $P$ value $=0.333)$. In the corticosteroid group as well as the laser group, there was a statistically significant decrease in pain scores post-treatment $(P=0.024, P=0.043$ respectively). Pair-wise comparisons revealed no statistically significant difference between post-treatment and follow-up periods; however, both post-treatment and follow-up period mean VAS scores were lower than pre-treatment scores (Table 2, Fig. 1).

\section{Clinical (RAE) score}

No statistically significant difference between the two groups was found before treatment $(P$ value $=0.096$ ) . After treatment, the corticosteroid group demonstrated lower mean RAE scores than the laser group which was statistically significant $(P$ value $=0.036)$. No statistically significant difference was seen between both in the follow-up period $(P$ value $=0.067)$. There was a statistically significant decrease in RAE post-treatment in both the corticosteroid and laser groups, $(P=0.004, P=$ 0.038 respectively). Pair-wise comparison revealed no statistical significant difference between post-treatment and follow-up periods; however, both groups demonstrated lower mean RAE than pre-treatment score (Table 3, Fig. 2).

\section{Improvement scores}

Better improvement results were seen in the steroid group where $50 \%$ of patients showed partial improvement, and the other $50 \%$ demonstrated total improvement. Only $16.7 \%$ of the patients showed total improvement in the laser group, with $66.7 \%$ demonstrating partial improvement and $16.7 \%$ demonstrating no improvement. However, no statistically significant difference was seen between the improvement parameters in the two groups $(P=0.117)$ Fig. 3.

\section{Discussion}

It is a therapeutic challenge to find the suitable treatment for the atrophic/erosive OLP disease, because although there are many medications for this potentially malignant disease, not all are successful in alleviating the symptoms of OLP lesions (Mahdavi et al. 2013; Misra et al. 2013).

In the present study, the efficacy of triamcinolone acetonide paste in reducing the signs and symptoms 
Table 2 Mean, standard deviation (SD) values, and results of comparison between VAS scores in the two groups as well as the changes within each group

\begin{tabular}{|c|c|c|c|c|c|}
\hline & \multicolumn{2}{|c|}{$\begin{array}{l}\text { Corticosteroids } \\
(n=12)\end{array}$} & \multicolumn{2}{|c|}{$\begin{array}{l}\text { Laser } \\
(n=12)\end{array}$} & \multirow{2}{*}{$\begin{array}{l}P \text { value } \\
\text { (between } \\
\text { groups) }\end{array}$} \\
\hline & Mean & SD & Mean & SD & \\
\hline \multicolumn{6}{|l|}{ Time } \\
\hline Pre-treatment & $6.8^{\mathrm{a}}$ & 0.9 & $7.0^{\mathrm{a}}$ & 1.8 & 0.807 \\
\hline Post-treatment & $0.9^{b}$ & 1.0 & $3.9^{\mathrm{b}}$ & 3.0 & $0.020^{*}$ \\
\hline Follow-up & $0.8^{b}$ & 1.0 & $1.5^{\mathrm{b}}$ & 0.7 & 0.333 \\
\hline$P$ value (within group) & \multicolumn{2}{|l|}{$0.024^{*}$} & \multicolumn{2}{|l|}{$0.043^{*}$} & \\
\hline
\end{tabular}

Different superscripts in the same column are statistically significantly different *Significant at $P \leq 0.05$

of OLP was compared to that of LLLT using diode laser.

Corticosteroids remain the most frequently and reliably used medications for the treatment of symptomatic OLP, where triamcinolone acetonide is the most common commercial topical steroid used. However, considering the chronic nature of the disease, patients may develop unwanted side effects or may be unresponsive or resistant to this long-term or often repeated type of treatment (Amirchaghmaghi et al. 2015).

Recently, LLLT has been used successfully for the treatment of OLP. In a prospective study, low-level laser irradiation given to unresponsive OLP patients demonstrated significant decrease in clinical signs and symptoms with no side effects observed (Cafaro et al. 2010). Also, 19 females who had OLP in their tongues reported reduction of pain, discomfort, and lesions' size after receiving LLLT (Cheng et al. 2012). In another study, more favorable clinical and symptomatic results were achieved in patients receiving LLLT in comparison to those treated with CO2 laser (Agha-Hosseini et al. 2012).

No statistical significant difference was seen between the corticosteroid and laser groups regarding age and gender in the present study population with a noticed

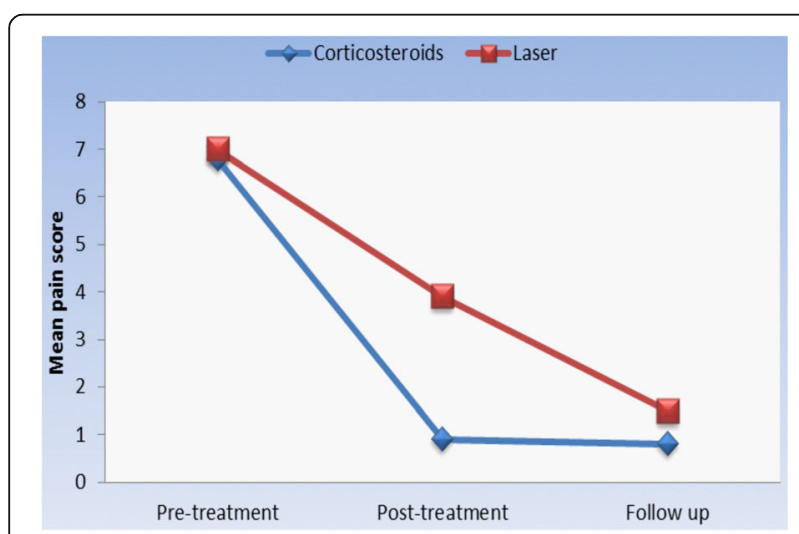

Fig. 1 Changes in mean pain (VAS) scores in the corticosteroid and laser groups
Table 3 Mean, standard deviation (SD) values, and results of comparison between RAE scores in the two groups as well as the changes within each group

\begin{tabular}{|c|c|c|c|c|c|}
\hline & \multicolumn{2}{|c|}{$\begin{array}{l}\text { Corticosteroids } \\
(n=12)\end{array}$} & \multicolumn{2}{|c|}{$\begin{array}{l}\text { Laser } \\
(n=12)\end{array}$} & \multirow{2}{*}{$\begin{array}{l}P \text { value } \\
\text { (between } \\
\text { groups) }\end{array}$} \\
\hline & Mean & SD & Mean & SD & \\
\hline \multicolumn{6}{|l|}{ Time } \\
\hline Pre-treatment & $19.9^{\mathrm{a}}$ & 8.9 & $27.5^{\mathrm{a}}$ & 10.7 & 0.096 \\
\hline Post-treatment & $6.3^{b}$ & 4.1 & $14.1^{\mathrm{b}}$ & 8.9 & $0.036^{*}$ \\
\hline Follow-up & $5.0^{\mathrm{b}}$ & 1.9 & $11.0^{b}$ & 2.9 & 0.067 \\
\hline$P$ value (within group) & \multicolumn{2}{|l|}{$0.004^{*}$} & \multicolumn{2}{|l|}{$0.038^{*}$} & \\
\hline
\end{tabular}

Different superscripts in the same column are statistically significantly different * Significant at $P \leq 0.05$

prevalence in females which agrees with other reports (McCartan and Healy 2008; Gupta and Jawanda 2015; Cafaro et al. 2010).

Diode laser $(980 \mathrm{~nm})$ possesses a deep power of penetration reaching about $1.5 \mathrm{~mm}$ (Cheng et al. 2012). Application of diode will increase the temperature of the affected tissues to above $50^{\circ}$ and less than $100^{\circ}$ causing blanching of the affected mucosal tissue and protein denaturation which in turn will destroy the affected epithelial tissues with its surface antigen (van der Hem et al. 2008). Re-epithelization occurs within 3 weeks after removal of the epithelium by the laser and any feeling of discomfort when in contact with food or liquid disappears (Fornaini 2012).

A $970-\mathrm{nm}$ diode laser was used in the present study for the treatment of OLP because from an optical view, a diode laser in this wavelength has a more superficial action than the 810- and the 904-nm laser which can be more beneficial in treating erosive and atrophic OLP lesions (Cafaro et al. 2014; Byrnes et al. 2005). Setting the exposure time at $4 \mathrm{~min}$ was decided after a pilot study on five patients, aiming at achieving max benefits with minimal post-exposure side effects. Patients received two laser sessions per week with a maximum of ten

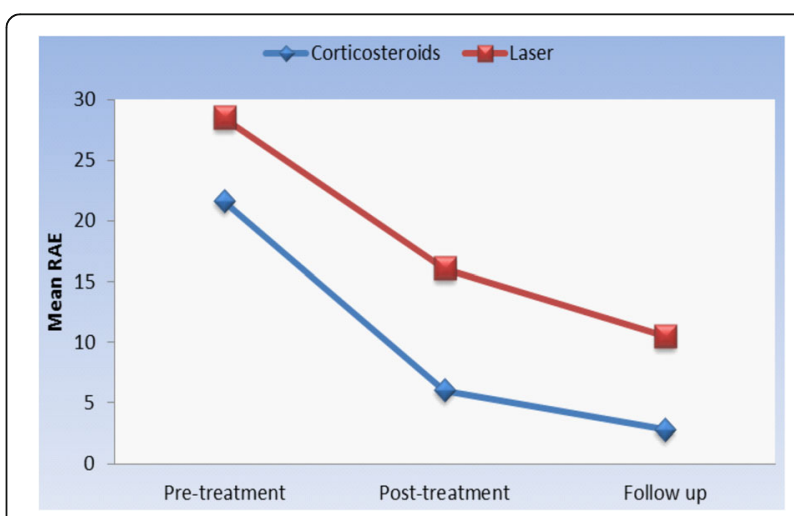

Fig. 2 Changes in mean RAE scores in the corticosteroid and laser groups 


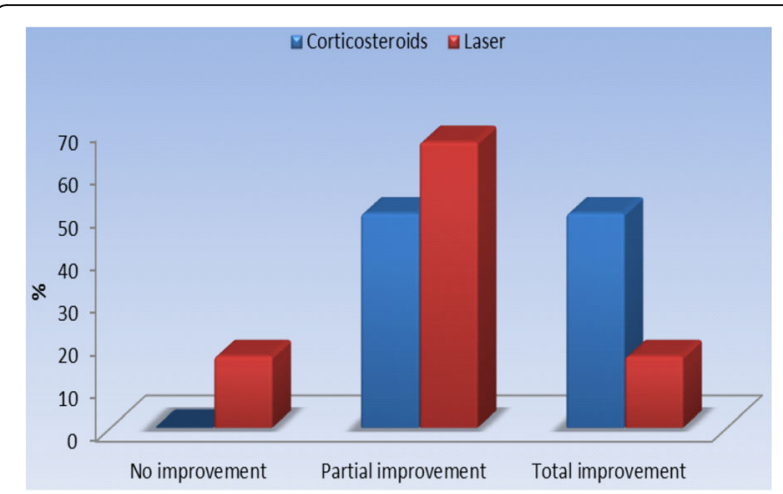

Fig. 3 Improvement percentage in the corticosteroid and laser groups

sessions which is in accordance with several previous studies (Cafaro et al. 2010; HH et al. 2011; Misra et al. 2013). Two watts of power was used since using higher power would decrease the cellular proliferation, thus slowing the healing process (Sattayut et al. 2013).

In our comparison, both treatment modalities demonstrated reduction of VAS and RAE scores; however, statistical analysis revealed lower mean VAS and RAE scores in the triamcinolone acetonide group when compared with those of the diode laser group. Moreover, $50 \%$ of the patients treated with steroids demonstrated total improvement, while only $16.7 \%$ of patients demonstrated total improvement in the laser group.

The lower VAS and RAE scores seen in the corticosteroid group can be attributed to the anti-inflammatory effect of steroids causing suppression of the T cell action, a function which is specific to the autoimmune nature of OLP where $\mathrm{T}$ lymphocytes play the major role in the pathogenesis of the disease (Gonzalez-Garcia et al. 2006; Xia et al. 2006).

On the other hand, LLLT as diode laser has a biostimulatory effect consisting of vasodilatation, increasing cellular proliferation, activation of fibroblasts, and neutrophils and decreasing the number of inflammatory mediators, thus aiding in resolution of inflammation and enhancing wound healing. Reduction of pain is caused by the ability of laser to alter the individual's pain threshold, enhance aggregation of beta-endorphins and encephalins in the tissues, and decrease the C-fiber activity (Misra et al. 2013; Shirani et al. 2009; Cavalcanti et al. 2011).

This biostimulatory mechanism of laser appears to be host-dependent (acting by stimulation of the reparative process of the individual) in opposition to the specific suppressing function of $\mathrm{T}$ cells of steroids, which might explain the better results reducing VAS, RAE scores, and the superior improvement seen in the steroid group compared to the laser group seen in this work. Our results can be compared to a prospective case series of 30 patients affected by OLP, who received a 980-nm diode laser 18 , where $60 \%$ of the patients had a total resolution and $33.3 \%$ a partial resolution and no resolution of the lesions at all in $6.6 \%$ of patients (van der Meij and van der Waal 2003). In the present work, no statistical significant difference was seen between the improvement parameters in the two groups, where $16.7 \%$ of the patients showed total improvement in the laser group, with $66.7 \%$ demonstrating partial improvement and 16.7\% demonstrating no improvement. The group treated with triamcinolone paste demonstrated 50\% partial improvement and 50\% total improvement in OLP patients. This is in agreement with a previous study where the OLP group treated with triamcinolone acetonide showed equal cases of clinical complete and partial remission (50\%) (Thongprasom et al. 2007). Also, another work comparing the efficacy of fluocinolone acetonide (FAO) with that of triamcinolone acetonide in the management of symptomatic OLP for 4 weeks revealed that 13 of 19 patients could be effectively cured with FAO whereas only 8 of 19 patients (50\%) were cured with triamcinolone acetonide, coinciding with the 50\% total improvement results seen in our study (Thongprasom et al. 1992). In a similar study comparing the effectiveness of topical steroid versus LLLT, $63 \%$ of patients using laser therapy had more than 50\% lesion improvement; however, laser therapy was as effective as topical corticosteroids with reduction of pain, appearance, and severity scores in both groups with no significant differences found between them (Akbulut et al. 2013), which is in accordance with the non-statistical difference seen in improvement parameters in our work.

\section{Conclusions}

Within the limitations of this study, it can be concluded that although topical steroids were more effective in reducing the pain and RAE scores in OLP patients than laser treatment, using LLLT may be considered as an alternative therapy for the symptomatic treatment for OLP especially where corticosteroids are contraindicated or in the case of patients' resistance or incompliance. Further trials with larger sample size and longer followup periods are recommended.

\section{Abbreviations \\ LLLT: Low-level laser therapy; OLP: Oral lichen planus; RAE: Reticular, atrophic, erosive; VAS: Visual analog scale}

\section{Funding}

The work was self-funded by the authors.

Availability of data and materials

Data on which the conclusions of this work were based on are all presented in the manuscript and its additional files. 


\section{Authors' contributions}

All authors have contributed significantly to the conception and design of study, acquisition and interpretation of data, the clinical work, and the drafting and revision of the manuscript. All authors read and approved the final manuscript.

\section{Ethics approval and consent to participate}

The study protocol was approved by the Ethical Committee of the National Research Centre (reference no: 15023). All patients were informed about the treatment plan and they all submitted a written informed consent before enrolment, which was carried out in accordance with the declaration of Helsinki.

\section{Consent for publication}

Not applicable.

\section{Competing interests}

The authors declare that they have no competing interests.

\section{Publisher's Note}

Springer Nature remains neutral with regard to jurisdictional claims in published maps and institutional affiliations.

Received: 4 July 2018 Accepted: 2 October 2018

Published online: 24 October 2018

\section{References}

Aggarwal H, Singh MP, Nahar P, Mathur H, Sowmya GV (2014) Efficacy of lowlevel laser therapy in treatment of recurrent Aphthous ulcers - a sham controlled split mouth follow up study. J Clin Diagn Res 8:218-221

Agha-Hosseini F, Moslemi E, Mirzaii-Dizgah I (2012) Comparative evaluation of low-level laser and $\mathrm{CO}_{2}$ laser in treatment of patients with oral lichen planus. Int J Oral Maxillofac Surg 41:1265-1269

Akbulut N, Kursun ES, Tumer MK, Kamburoglu K, Gulsen U (2013) Is the 810-nm diode laser the best choice in oral soft tissue therapy? Eur J Dent 7:207-211

Amirchaghmaghi M, Delavarian Z, Iranshahi M, Shakeri MT, Mosannen Mozafari P, Mohammad pour AH, Farazi F, Iranshahy M (2015) A randomized placebocontrolled double blind clinical trial of quercetin for treatment of oral lichen planus. J Dent Res Dent Clin Dent Prospects 9:23-28

Arunkumar S, Kalappanavar AN, Annigeri RG, Kalappa SG (2015) Relative efficacy of pimecrolimus cream and triamcinolone acetonide paste in the treatment of symptomatic oral lichen planus. Indian J Dent 6:14-19

Byrnes KR, Waynant RW, Ilev IK, Wu X, Barna L, Smith K, Heckert R, Gerst H, Anders JJ (2005) Light promotes regeneration and functional recovery and alters the immune response after spinal cord injury. Laser Surg Med 36:171-185

Cafaro A, Albanese G, Arduino PG, Mario C, Massolini G, Mozzati M et al (2010) Effect of low-level laser irradiation on unresponsive oral lichen planus: early preliminary results in 13 patients. Photomed Laser Surg 28:S99-S103

Cafaro A, Arduino PG, Massolini G, Romagnoli E, Broccoletti R (2014) Clinical evaluation of the efficiency of low-level laser therapy for oral lichen planus: a prospective case series. Lasers Med Sci 29:185-190

Canto AM, Müller H, Freitas RR, Santos PS (2010) Oral lichen planus (OLP): clinical and complementary diagnosis. An Bras Dermatol 85:669-675

Cavalcanti TM, Almeida-Barros RQ, Catão MH, Feitosa AP, Lins RD (2011) Knowledge of the physical properties and interaction of laser with biological tissue in dentistry. An Bras Dermatol 86:955-960

Cheng S, Kirtschig G, Cooper S, Thornhill M, Leonardi-Bee J, Murphy R (2012) Interventions for erosive lichen planus affecting mucosal sites. Cochrane Database Syst Rev 15:CD008092

Fornaini C (2012) LLLT in the symptomatic treatment of oral lichen planus. Laser Ther 21:51-53

Gonzalez-Garcia A, Diniz-Freitas M, Gandara-Vila P, Blanco-Carrion A, Garcia-Garcia A, Gandara-Rey J (2006) Triamcinolone acetonide mouth rinses for treatment of erosive oral lichen planus: efficacy and risk of fungal over-infection. Oral Dis 12:559-565

Gupta S, Jawanda MK (2015) Oral lichen planus: an update on etiology, pathogenesis, clinical presentation, diagnosis and management. Indian J Dermat 60:222-229
HH J, Falaki F, Mahdavi O (2011) A comparative pilot study of low intensity laser versus topical corticosteroids in the treatment of erosive-atrophic oral lichen planus. Photomed Laser Surg 29:421-425

Jin JY, Lee SH, Yoon HJ (2010) A comparative study of wound healing following incision with a scalpel, diode laser or Er, Cr:YSGG laser in guinea pig oral mucosa: a histological and immunohistochemical analysis. Acta Odontol Scand 68:232-238

Kaplan I, Ventura-Sharabi Y, Gal G, Calderon S, Anavi Y (2012) The dynamics of oral lichen planus: a retrospective clinicopathological study. Head Neck Pathol 6:178-183

Mahdavi O, Boostani N, Jajarm H, Falaki F, Tabesh A (2013) Use of low level laser therapy for oral lichen planus: report of two cases. J Dent (Shiraz) 14:201-204

McCartan BE, Healy CM (2008) The reported prevalence of oral lichen planus: a review and critique. J Oral Pathol Med 37:447-453

Misra N, Chittoria N, Umapathy D, Misra P (2013) Efficacy of diode laser in the management of oral lichen planus. BMJ Case Rep 15

Rodríquez-Pérez M, Bravo-Pérez M, Sánchez-López JD, Muñoz-Soto E, RomeroOlid MN, Baca-García P (2013) Effectiveness of 1\% versus $0.2 \%$ chlorhexidine gels in reducing alveolar osteitis from mandibular third molar surgery: a randomized, double-blind clinical trial. Med Oral Patol Oral Cir Bucal 18: e693-e700

Sattayut S, Trivibulwanich J, Pipithirunkarn N, Danvirutai N (2013) A clinical efficacy of using $\mathrm{CO} 2$ laser irradiating to transparent gel on aphthous stomatitis patients. Laser Ther. 22:283-289

Shirani AM, Gutknecht N, Taghizadeh M, Mir M (2009) Low level laser therapy and myofacial pain dysfunction syndrome: a randomized controlled clinical trial. Lasers Med Sci 24:715-720

Sousa FA, Rosa LE (2008) Oral lichen planus: clinical and histopathological considerations. Braz J Otorhinolaryngol 74:284-292

Thongprasom K, Chaimusig M, Korkij W, Sererat T, Luangjarmekorn L, Rojwattanasirivej S (2007) A randomized-controlled trial to compare topical cyclosporin with triamcinolone acetonide for the treatment of oral lichen planus. J Oral Pathol Med 36:142-146

Thongprasom K, Luang Jarmekorn L, Seretat T, Taweesap W (1992) Relative efficacy of fluocinolone acetonide compared with triamcinolone acetonide in the treatment of oral lichen planus. J Oral Pathol Med. 21:456-458

van der Hem PS, Egges M, van der Wal JE, Roodenburg JL (2008) CO2 laser evaporation of oral lichen planus. Int J Oral Maxillofac Surg 37:630-633

van der Meij EH, van der Waal I (2003) Lack of clinicopathologic correlation in the diagnosis of oral lichen planus based on the presently available diagnostic criteria and suggestions for modifications. J Oral Pathol Med. 32:507-512

Vickers AJ (2006) How to randomize. J Soc Integr Oncol 4:194-198 Review Xia J, Li C, Hong Y, Yang L, Huang Y, Cheng B (2006) Short-term clinical evaluation of intralesional triamcinolone acetonide injection for ulcerative oral lichen planus. J Oral Pathol Med. 35:327-331

\section{Submit your manuscript to a SpringerOpen ${ }^{\circ}$ journal and benefit from:}

- Convenient online submission

- Rigorous peer review

- Open access: articles freely available online

- High visibility within the field

- Retaining the copyright to your article

Submit your next manuscript at $\boldsymbol{\nabla}$ springeropen.com 